\title{
FOURIER-LAPLACE TRANSFORMS AND THE BERGMAN SPACES
}

\author{
SABUROU SAITOH
}

(Communicated by Irwin Kra)

\begin{abstract}
The Fourier-Laplace transforms on $R^{n}(n \geq 2)$ whose images belong to the Bergman spaces are investigated from the point of view of a general theory of integral transforms. The central problems are to give the expressions of the Bergman kernels in terms of the Fourier-Laplace transforms, and to investigate the relationship between the domains and the ranges in the expressions.
\end{abstract}

1. Introduction. We let $G$ be a domain in $R^{n}$ and let $T_{G}=R^{n}+i G \subset \mathbf{C}^{n}$ be the tube domain over $G$. Then, T. G. Genchev $[6,7]$ discussed the Fourier-Laplace transforms when the images belong to the Bergman spaces on $T_{G}$ in contrast with the classical cases of the analytic Hardy and Szegö spaces. See Fuks [4], Gindikin $[\mathbf{8}, \mathbf{9}]$, and Stein and Weiss [17] for the classical cases. In this paper, following the general theory $[12, \mathbf{1 3}, \mathbf{1 4}]$ of integral transforms we will discuss the FourierLaplace transforms when the images belong to the Bergman spaces. Then, we will be able to obtain some general and unified results. The case of one-dimensional spaces was discussed in $[\mathbf{6}, \mathbf{1 5}]$.

2. Isometrical identities and inverse formulas. Following the general theory of integral transforms, we first design our situation for the Fourier-Laplace transforms from the point of view that the images belong to the Bergman spaces.

We set

$$
\begin{aligned}
z & =\left(z_{1}, z_{2}, \ldots, z_{n}\right) \in \mathbf{C}^{n}, \quad z_{j}=x_{j}+y_{j}\left(x_{j}, y_{j} \in R\right) \\
x & =\left(x_{1}, x_{2}, \ldots, x_{n}\right), \quad y=\left(y_{1}, y_{2}, \ldots, y_{n}\right) \\
t & =\left(t_{1}, t_{2}, \ldots, t_{n}\right) \in R^{n}
\end{aligned}
$$

and

$$
(z, t)=\sum_{j=1}^{n} z_{j} t_{j} .
$$

For some domains $D \subset R^{n}, \mathscr{R} \subset \mathbf{C}^{n}$ and for some functions $g$, we consider the Fourier-Laplace transform

$$
f(z)=\frac{1}{(2 \pi)^{n / 2}} \int_{D} e^{i(z, t)} g(t) d t, \quad z \in \mathscr{R} .
$$

Received by the editors January 15, 1987.

1980 Mathematics Subject Classification (1985 Revision). Primary 30C40; Secondary 32A07, $42 \mathrm{~B} 10$.

Key words and phrases. Fourier-Laplace transform, Bergman kernel, tube domain, convex domain, general theory of integral transforms.

Research partially supported by Grant-in-Aid for Scientific Research 60540086. 
When we discuss the case that the images $f(z)$ belong to the Bergman space $\operatorname{Berg}(\mathscr{R})$, then we should consider the following expression:

For the Bergman kernel $K(z, \bar{w} ; \mathscr{R})$ on $\mathscr{R}$ and a positive continuous function $\rho(t)$ on $D$

$$
K(z, \bar{w} ; \mathscr{R})=\frac{1}{(2 \pi)^{n}} \int_{D} e^{i(z, t)} e^{-i(\bar{w}, t)} \rho(t) d t .
$$

See the general theory $[\mathbf{1 2}, \mathbf{1 3}, \mathbf{1 4}]$ of integral transforms for this idea. The space $\operatorname{Berg}(\mathscr{R})$ is composed of all holomorphic functions $f(z)$ on $\mathscr{R}$ which are integrable on $\mathscr{R}$ and with finite norms $\left\{\int_{\mathscr{R}}|f(z)|^{2} d x d y\right\}^{1 / 2}<\infty$. The kernel $K(z, \bar{w} ; \mathscr{R})$ is the reproducing kernel for the space $\operatorname{Berg}(\mathscr{R})$. See Bochner and Martin [3] and Fuks [5] for this space and kernel.

Since the existence domain of the Bergman kernel $K(z, \bar{w} ; \mathscr{R})$ in $(2.2)$ is independent of $x$, we immediately see that we should take the tube domain $T_{G}$ over $G$ as $\mathscr{R}$.

We will determine the weight $\rho(t)$. From (2.1), by Parseval's equation we have

$$
\int_{R^{n}}|f(z)|^{2} d x=\int_{D} e^{-2(y, t)}|g(t)|^{2} d t
$$

and so, formally,

$$
\int_{T_{G}}|f(z)|^{2} d x d y=\int_{D}|g(t)|^{2}\left\{\int_{G} e^{-2(y, t)} d y\right\} d t .
$$

When (2.3) is valid, then for

$$
W(t ; G)=\int_{G} e^{-2(y, t)} d y
$$

we obtain the isometrical identity

$$
\int_{T_{G}}|f(z)|^{2} d x d y=\int_{D}|g(t)|^{2} W(t ; G) d t .
$$

As we see from the general theory of integral transforms, the above arguments will mean that the Bergman kernel $K\left(z, \bar{w} ; T_{G}\right)$ has the expression

$$
K\left(z, \bar{w} ; T_{G}\right)=\frac{1}{(2 \pi)^{n}} \int_{D} e^{i(z, t)} e^{-i(\bar{w}, t)} W(t ; G)^{-1} d t
$$

for some domain $D$. Further, then in the integral transform

$$
f(z)=\frac{1}{(2 \pi)^{n}} \int_{D} F(t) e^{i(z, t)} W(t ; G)^{-1} d t
$$

for functions $F$ satisfying

$$
\int_{D}|F(t)|^{2} W(t ; G)^{-1} d t<\infty
$$

we will have the isometrical identity

$$
\int_{T_{G}}|f(z)|^{2} d x d y=\frac{1}{(2 \pi)^{n}} \int_{D}|F(t)|^{2} W(t ; G)^{-1} d t .
$$

Conversely, we will determine the condition that the Bergman kernel $K\left(z, \bar{w} ; T_{G}\right)$ is expressible in the form (2.5). For this problem, we obtain the following fundamental theorem. 
THEOREM 2.1. Integral (2.5) exists on $T_{G} \times \overline{T_{G}}$ (complex conjugate) and the function $K\left(z, \bar{w} ; T_{G}\right)$ defined by (2.5) is the Bergman kernel on $T_{G}$ if and only if

$$
D=\hat{D}_{G}=\left\{t \in R^{n} ; W(t ; G)<\infty\right\} ;
$$

that is, $\hat{D}_{G}$ is the maximal domain satisfying $W(t ; G)<\infty$.

ProOF. We first note that $\hat{D}_{G}$ is a convex domain on $R^{n}$, as we see from the inequality

$$
u^{\alpha} v^{1-\alpha} \leq \alpha u+(1-\alpha) v \quad(u, v \geq 0,0<\alpha<1) .
$$

We assume that (2.9) is valid. Then, for any $y^{(0)} \in G$, we take a neighborhood such that

$$
U\left(y^{(0)}, \varepsilon\right)=\prod_{j=1}^{n}\left(y_{j}^{(0)}-\varepsilon, y_{j}^{(0)}+\varepsilon\right) \subset G
$$

for some $\varepsilon>0$. Then, we have

$$
\begin{aligned}
\int_{\hat{D}_{G}} & e^{-2\left(y^{(0)}, t\right)} W(t ; G)^{-1} d t \\
& \leq \int_{\hat{D}_{G}} e^{-2\left(y^{(0)}, t\right)} \frac{d t}{\int_{U\left(y^{(0)}, \varepsilon\right)} e^{-2(y, t)} d y} \\
& \leq \int_{R^{n}} e^{-2\left(y^{(0)}, t\right)} \frac{d t}{\prod_{j=1}^{n}\left(1 / 2 t_{j}\right)\left\{\exp \left[-2\left(y_{j}^{(0)}-\varepsilon\right) t_{j}\right]-\exp \left[-2\left(y_{j}^{(0)}+\varepsilon\right) t_{j}\right]\right\}} \\
& \leq 2^{n} \prod_{j=1}^{n} \int_{-\infty}^{\infty} \exp \left(-2 y_{j}^{(0)} t_{j}\right) \frac{t_{j}}{\exp \left[-2\left(y_{j}^{(0)}-\varepsilon\right) t_{j}\right]-\exp \left[-2\left(y_{j}^{(0)}+\varepsilon\right) t_{j}\right]} d t_{j} \\
& <\infty
\end{aligned}
$$

Hence, the function $K\left(z, \bar{w} ; T_{G}\right)$ defined by $(2.5)$ on $\hat{D}_{G}$ exists on $T_{G} \times \overline{T_{G}}$. Recall here also the theorem of Sommer and Mehring [16] (see Fuks [5, p. 85]).

Since the function $K\left(z, \bar{w} ; T_{G}\right)$ is a positive matrix on $T_{G}$ in the sense of E. M. Moore, the Hilbert space $H\left(T_{G}, \hat{D}_{G}\right)$ composed of functions on $T_{G}$ admitting the reproducing kernel $K\left(z, \bar{w} ; T_{G}\right)$ exists uniquely (see Aronszajn $[\mathbf{1}, \mathbf{2}]$ ). This Hilbert space $H\left(T_{G}, \hat{D}_{G}\right)$ is composed of all holomorphic functions $f(z)$ on $T_{G}$ which are expressible in the form

$$
f(z)=\frac{1}{(2 \pi)^{n}} \int_{\hat{D}_{G}} F(t) e^{i(z, t)} W(t ; G)^{-1} d t
$$

for the Hilbert space $L_{2}\left(\hat{D}_{G}, W(t ; G)^{-1} d t\right)$ composed of all functions on $\hat{D}_{G}$ with finite norms

$$
\left\{\frac{1}{(2 \pi)^{n}} \int_{\hat{D}_{G}}|F(t)|^{2} W(t ; G)^{-1} d t\right\}^{1 / 2}<\infty
$$

and with the norm

$$
\|f\|_{H\left(T_{G}, \text { hat } D_{G}\right)}^{2}=\frac{1}{(2 \pi)^{n}} \int_{\hat{D}_{G}}|F(t)|^{2} W(t ; G)^{-1} d t
$$

$[12,13,14]$ 
Meanwhile, from (2.11) and Parseval's equation, we have as in (2.3)

$$
\int_{T_{G}}|f(z)|^{2} d x d y=\frac{1}{(2 \pi)^{n}} \int_{\hat{D}_{G}}|F(t)|^{2} W(t ; G)^{-1} d t .
$$

This implies that the Hilbert space $H\left(T_{G}, \hat{D}_{G}\right)$ is a subspace of the Bergman space $\operatorname{Berg}\left(T_{G}\right)$.

On the other hand, for any $f \in \operatorname{Berg}\left(T_{G}\right)$, we set

$$
\tilde{g}(t)=\frac{1}{(2 \pi)^{n / 2}} \int_{R^{n}} f(z) e^{-i(z, t)} d x .
$$

Of course, this integral exists and, further, $\tilde{g}$ is independent of $y \in G$ as we see from the Cauchy integral formula. See [17, pp. 98-101] for this argument. In addition, $\tilde{g}(t)$ is continuous (see [7]). Then, by Parseval's equation we have

$$
\int_{T_{G}}|f(z)|^{2} d x d y=\int_{G} \int_{R^{n}} e^{-2(y, t)}|\tilde{g}(t)|^{2} d t d y .
$$

Hence, in particular, a.e. on the support of $\tilde{g}, W(t ; G)<\infty$, and so, on the support of $\tilde{g}, W(t ; G)<\infty$. Thus, we see that any $f \in \operatorname{Berg}\left(T_{G}\right)$ is expressible in the form (2.11) with (2.12) and

$$
\tilde{g}(t)=\left\{\begin{array}{l}
\frac{1}{(2 \pi)^{n / 2}} F(t) W(t ; G)^{-1} \text { on } \hat{D}_{G}, \\
0 \text { on } R^{n}-\hat{D}_{G} .
\end{array}\right.
$$

Therefore, we have the desired relation $H\left(T_{G}, \hat{D}_{G}\right)=\operatorname{Berg}\left(T_{G}\right)$.

The necessity in Theorem 2.1 is clear, from the previous arguments of Theorem 2.1.

From the general theory of integral transforms and Theorem 2.1, we have

THEOREM 2.2. For the domain $\hat{D}_{G}$ satisfying (2.9) and for the integral transform (2.11) satisfying (2.12), we have the isometrical identity

$$
\|f\|_{\operatorname{Berg}\left(T_{G}\right)}^{2}=\frac{1}{(2 \pi)^{n}} \int_{\hat{D}_{G}}|F(t)|^{2} W(t ; G)^{-1} d t
$$

between the whole spaces $L_{2}\left(\hat{D}_{G}, W(t ; G)^{-1} d t\right)$ and $\operatorname{Berg}\left(T_{G}\right)$. Moreover, we have the inverse formula

$$
F(T) W(t ; G)=s-\lim \int_{N \rightarrow \infty} f(z) e^{-i(\bar{z}, t)} d x d y
$$

in the sense of the strong convergence in the space $L_{2}\left(\hat{D}_{G}, W(t ; G)^{-1} d t\right)$. Here, $\left\{E_{N}\right\}_{N=1}^{\infty}$ is any compact exhaustion of $T_{G}$.

3. The existence domains. For a given $\hat{D}_{G}$, we take the maximal domain in the sense that

$$
\hat{G}=\left\{y \in R^{n}: \int_{\hat{D}_{G}} e^{-2(y, t)} W(t ; G)^{-1} d t<\infty\right\}
$$

or

$$
\hat{G}=\left\{y \in R^{n} ; K\left(z, \bar{z} ; T_{G}\right)<\infty\right\}
$$


Then, we see that $\hat{G}$ is also convex. This fact corresponds to the classical fact that every holomorphic function in the tube domain $T_{G}$ has an analytic continuation to a holomorphic function on $T_{G^{c}}$, where $G^{c}$ is the convex hull of $G$. (See Bochner and Martin [3] and Fuks [5].) In our case, we can prove that $\hat{G}=G^{c}$, directly and any member $f$ of $\operatorname{Berg}\left(T_{G}\right)$ can be continued analytically onto $T_{\hat{G}}$ and its continuation belongs to $\operatorname{Berg}\left(T_{\hat{G}}\right)$.

When $G$ or $\hat{G}$ contains a whole line, then we see that the corresponding domain $\hat{D}_{G}$ or $\hat{D}_{\hat{G}}$ is void. This fact implies that: A necessary and sufficient condition for $\operatorname{Berg}\left(T_{G}\right)$ or $\operatorname{Berg}\left(T_{\hat{G}}\right)$ to contain a function that is not identically zero is that $G$ or $\hat{G}$ does not contain a whole line, respectively. (Cf. Stein and Weiss [17, p. 94].)

We will assume that $\hat{G}$ is bounded by a piecewise smooth hypersurface. Let $O$ be the origin of coordinates in the $y$-space $R^{n}$, and let the hyperplanes $\{\Gamma\}$ pass through it and lie parallel to the limiting positions of the tangent hyperplanes of the hypersurface. We consider the convex cone with vertex at the origin enveloped by these hyperplanes. The nappe of this cone lying on the same side of the hyperplanes $\{\Gamma\}$ as the domain $\hat{G}$ is called the asymptotic cone of the domain $T_{\hat{G}}$. As the asymptotic cone of a domain $T_{\hat{G}}$ with a bounded base $\hat{G}$, we take the set $\{O\}$, the origin of coordinates. Further, if $V$ is the asymptotic cone of $T_{\hat{G}}$, we will say that this domain $T_{\hat{G}}$ is of type $V$.

Let $\Gamma\left(\subset R^{n}\right)$ be a convex cone with vertex at the origin. Then, let $\Gamma^{*}$ be the conjugate cone of $\Gamma$, that is, the set defined by

$$
\Gamma^{*}=\left\{y^{*} \in R^{n} ;\left(y^{*}, y\right)>0 \text { for all } y \in \bar{\Gamma} \text { (closure), } y \neq 0\right\} .
$$

(See Fuks [4] for these definitions.) Then, we obtain

THEOREM 3.1. (I) When $\hat{G}$ is a bounded domain in $R^{n}$, we have $\hat{D}_{\hat{G}}=R^{n}$. (II) When $\hat{G}$ is a convex cone $\Gamma$ with vertex at the origin, we have $\hat{D}_{\hat{G}}=\Gamma^{*}$. (III) When $T_{\hat{G}}$ is of type $V$, we have $\hat{D}_{\hat{G}}=V^{*}$.

PROOF. (I) is clear and we see (II) from the following proof of (III).

For any fixed $t^{(0)} \in V^{*}$, we set

$$
\left(y, t^{(0)}\right)=|y|\left|t^{(0)}\right| \cos \vartheta_{0}(y)>0 \quad \text { on } V,
$$

where, of course, $\vartheta_{0}(y)\left(\left|\vartheta_{0}(y)\right|<\pi / 2\right)$ is the angle between the two vectors $y$ and $t^{(0)}$ in the same space $R^{n}$. Hence, there exists $\Theta$ such that

$$
\left|\vartheta_{0}(y)\right|<\Theta<\pi / 2 \text { on } V \text {. }
$$

Hence, there exist $\varepsilon>0$ and $M>0$ such that

$$
\left|\vartheta_{0}(y)\right|<\Theta-\varepsilon<\pi / 2 \text { on } \hat{G} \cap\{|y| \geq M\} .
$$

Then, from the identity

$$
\int_{|x| \leq N} f(|x|) d x=\frac{2 \sqrt{\pi^{n}}}{\Gamma(n / 2)} \int_{0}^{N} x^{n-1} f(x) d x
$$


$[10$, p. 623], we have

$$
\begin{aligned}
\int_{\hat{G}} e^{-2\left(y, t^{(0)}\right)} d y \leq \int_{\hat{G} \cap\{y \leq M\}} \exp \left[-2\left(y, t^{(0)}\right)\right] d y \\
\quad+\int_{\hat{G} \cap\{|y| \geq M\}} \exp \left[-2|y|\left|t^{(0)}\right| \cos (\Theta-\varepsilon)\right] d y \\
\leq \int_{\hat{G} \cap\{|y| \leq M\}} \exp \left[-2\left(y, t^{(0)}\right)\right] d y \\
\quad+\int_{R^{n}} \exp \left[-2|y|\left|t^{(0)}\right| \cos (\Theta-\varepsilon)\right] d y \\
\leq \int_{\hat{G} \cap\{|y| \leq M\}} \exp \left[-2\left(y, t^{(0)}\right)\right] d y \\
\quad+\lim _{N \rightarrow \infty} \frac{2 \sqrt{\pi^{n}}}{\Gamma(n / 2)} \int_{0}^{N} y^{n-1} \exp \left[-y\left(2\left|t^{(0)}\right| \cos (\Theta-\varepsilon)\right)\right] d y
\end{aligned}
$$

Hence, we have $V^{*} \subset \hat{D}_{\hat{G}}$.

On the other hand, for any $R^{n}-V^{*} \ni t^{(0)}$, by definition of $V^{*}$, there exists a point $y^{(0)} \in V$ such that $\left(y^{(0)}, t^{(0)}\right)<0$. Then, there exists a narrow nondegenerate (i.e. contains an $n$-dimensional sphere) convex cone $\Gamma\left(y^{(0)}\right)$ with vertex at the origin such that $\left(y, t^{(0)}\right)<0$ on $\Gamma\left(y^{(0)}\right)$ and

$$
\hat{G} \supset \Gamma\left(y^{(0)}\right) \cap\{|y| \geq M\} \text { for some constant } M>0 .
$$

Then, we have

$$
\begin{aligned}
\int_{\hat{G}} e^{-2\left(y, t^{(0)}\right)} d y & \geq \int_{\Gamma\left(y^{(0)}\right) \cap\{|y| \geq M\}} e^{-2\left(y, t^{(0)}\right)} d y \\
& \geq \int_{\Gamma\left(y^{(0)}\right) \cap\{|y| \geq M\}} d y=\infty .
\end{aligned}
$$

Hence, $V^{*} \supset \hat{D}_{\hat{G}}$, and so we have the desired assertion $V^{*}=\hat{D}_{\hat{G}}$.

EXAMPLES. (I) When $\hat{G}=\{(-a, a) \times(-a, a) \times \cdots \times(-a, a)\} \subset R^{n}, \hat{D}_{\hat{G}}=R^{n}$. Further,

$$
W(t ; \hat{G})=\prod_{j=1}^{n} \int_{-a}^{a} \exp \left(-2 y_{j} t_{j}\right) d y_{j}=\prod_{j=1}^{n} \frac{1}{t_{j}} \sinh 2 a t_{j} .
$$

Hence, from the identity

$$
\frac{1}{(2 \pi)^{1 / 2}} \int_{-\infty}^{\infty} \frac{x}{\sinh x} e^{i \xi x} d x=\frac{(2 / \pi \xi)^{1 / 2} e^{\pi \xi}}{\left(1+e^{\pi \xi}\right)^{2}}
$$

[10, p. 1148], we have

$$
K\left(z, \bar{z} ; T_{G}\right)=\left(\frac{1}{16 a^{2} \pi^{2}}\right)^{n} \prod_{j=1}^{n} \operatorname{sech}^{2} \frac{\pi y_{j}}{2 a}
$$

and so

$$
K\left(z, \bar{w} ; T_{G}\right)=\left(\frac{1}{16 a^{2} \pi^{2}}\right)^{n} \prod_{j=1}^{n} \operatorname{sech}^{2} \frac{\pi\left(z_{j}-\bar{w}_{j}\right)}{4 a \dddot{\imath}}
$$


(II) When $\hat{G}$ is the octant $\left\{y_{1}>0, \ldots, y_{n}>0\right\} \subset R^{n}, T_{\hat{G}}$ is of type $V=\hat{G}$ and $\hat{D}_{\hat{G}}=V^{*}=\hat{G}$. Then,

$$
W(t ; \hat{G})=\frac{1}{2^{n}} \prod_{j=1}^{n} \frac{1}{t_{j}}
$$

Hence,

$$
K\left(z, \bar{z} ; T_{G}\right)=\frac{1}{4^{n} \pi^{n}} \prod_{j=1}^{n} \frac{1}{y_{j}^{2}}
$$

and so

$$
K\left(z, \bar{w} ; T_{G}\right)=\frac{(-1)^{n}}{\pi^{n}} \prod_{j=1}^{n} \frac{1}{\left(z_{j}-\bar{w}_{j}\right)^{2}} .
$$

(III) When $\hat{G}=\left\{y_{2}>e^{-y_{1}}\right\} \subset R^{2}, T_{\hat{G}}$ is of type $V=\left\{y_{1}>0, y_{2}>0\right\}$. Then, $\hat{D}_{\hat{G}}=V^{*}=V$, and

$$
W(t ; \hat{G})=\frac{1}{2 t_{2}} \int_{0}^{\infty} \exp \left[-2\left(y_{1} t_{1}+e^{-y_{1}} t_{2}\right)\right] d y_{1} .
$$

Certainly, for any $t \in V, W(t ; \hat{G})<\infty$.

It is, in general, difficult to compute the Bergman kernel $K\left(z, \bar{w} ; T_{G}\right)$ concretely, but for the concrete expressions of the Bergman kernels of the classical domains, see $\mathrm{Hau}[\mathbf{1 1}]$ and Gindikin $[\mathbf{8}, \mathbf{9}]$.

We will be able to discuss the weighted Bergman kernel on $T_{G}$ and inequalities of Hausdorff-Young type for general $L_{p}\left(D, W(t ; G)^{-1} d t\right)(p \geq 1)$ functions as in [15]. But, the arguments are similar and formal, and we do not refer to them here.

\section{REFERENCES}

1. N. Aronzsajn, La théorie des noyaux reproduisants et ses applications. I. Proc. Cambridge Philos. Soc. 39 (1943), 113-153.

2. __ Theory of reproducing kernels, Trans. Amer. Math. Soc. 68 (1950), 337-404.

3. S. Bochner and W. T. Martin, Several complex variables, Princeton Univ. Press, Princeton, N.J., 1948.

4. B. A. Fuks, Introduction to the theory of analytic functions of several complex variables, Transl. Math. Mono., vol. 8, Amer. Math. Soc., Providence, R.I., 1963.

5. _ Special chapters in the theory of analytic functions of several complex variables, Transl. Math. Mono., vol. 14, Amer. Math. Soc., Providence, R.I., 1965.

6. T. G. Genchev, Some theorems of Paley-Wiener's type, Complex Analysis and Applications 83, Sofia, 1985.

7. __ Paley-Wiener type theorem for functions in Bergman spaces over tube domains, J. Anal. Appl. (to appear).

8. S. G. Gindikin, Integral formulas for Siegel domains of the second kind, Soviet Math. Dokl. 2 (1961), 1480-1483.

9. __ Analytic functions in tubelar regions, Soviet Math. Dokl. 3 (1962), 1178-1183.

10. I. S. Gradshleyn and I. M. Ryzhik, Tables of integrals, series and product, Academic Press, New York, 1980.

11. L. K. Hua, Harmonic analysis of functions of several complex variables in the classical domains, Transl. Math. Mono., vol. 6, Amer. Math. Soc., Providence, R.I., 1963.

12. S. Saitoh, Integral transforms in Hilbert spaces, Proc. Japan Acad. 58 (1982), 361-364. 
13. _ Hilbert spaces induced by Hilbert space valued functions, Proc. Amer. Math. Soc. 89 (1983), 74-78.

14. _ A general theory of integral transforms and its applications, Math. Vesnik 37 (1985), 121-133.

15. _ The Laplace transform of $L_{p}$ functions with weights, Appl. Anal. 22 (1986), 103-109.

16. F. Sommer and J. Mehring, Kernfunktion and Hüllenbildung in der Funktionen theorie vonmehrerer Veränderlichen, Math. Ann. 131 (1956), 1-16.

17. E. M. Stein and G. Weiss, Introduction to Fourier analysis on Euclidean spaces, Princeton Univ. Press, Princeton, N.J., 1975.

Department of Mathematics, Faculty of Engineering, Gunma University, KIRYU 376, JAPAN 